\title{
Proximity Effects on the Charge Density Wave Order and Superconductivity in Single-Layer $\mathrm{NbSe}_{2}$
}

Paul Dreher, ${ }^{\nabla}$ Wen Wan, ${ }^{\nabla}$ Alla Chikina, Marco Bianchi, Haojie Guo, Rishav Harsh, Samuel Mañas-Valero, Eugenio Coronado, Antonio J. Martínez-Galera, Philip Hofmann, Jill A. Miwa,* and Miguel M. Ugeda*

Cite This: ACS Nano 2021, 15, 19430-19438

Read Online

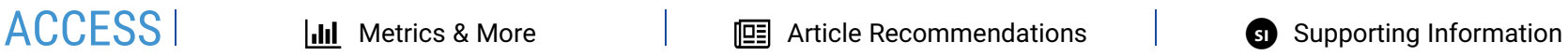

ABSTRACT: Collective electronic states such as the charge density wave (CDW) order and superconductivity (SC) respond sensitively to external perturbations. Such sensitivity is dramatically enhanced in two dimensions (2D), where 2D materials hosting such electronic states are largely exposed to the environment. In this regard, the ineludible presence of supporting substrates triggers various proximity effects on 2D materials that may ultimately compromise the stability and properties of the electronic ground state. In this work, we investigate the impact of proximity effects on the CDW and superconducting states in single-layer (SL) $\mathrm{NbSe}_{2}$ on four
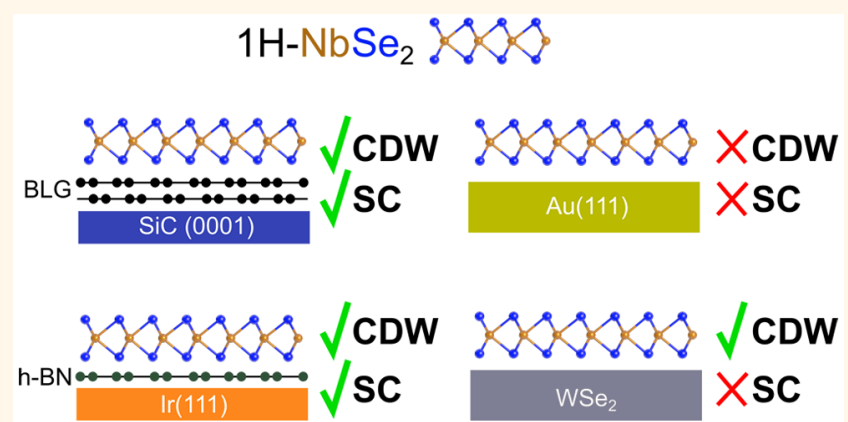
substrates of diverse nature, namely, bilayer graphene (BLG), SL-boron nitride (h-BN), Au(111), and bulk WSe ${ }_{2}$. By combining low-temperature (340 mK) scanning tunneling microscopy/ spectroscopy and angle-resolved photoemission spectroscopy, we compare the electronic structure of this prototypical 2D superconductor on each substrate. We find that, even when the electronic band structure of SL-NbSe ${ }_{2}$ remains largely unaffected by the substrate except when placed on $\mathrm{Au}(111)$, where a charge transfer occurs, both the CDW and SC show disparate behaviors. On the insulating h-BN/Ir(111) substrate and the metallic BLG/SiC(0001) substrate, both the $3 \times 3$ CDW and superconducting phases persist in SL-NbSe ${ }_{2}$ with very similar properties, which reveals the negligible impact of graphene on these electronic phases. In contrast, these collective electronic phases are severely weakened and even absent on the bulk insulating WSe $\mathrm{C}_{2}$ substrate and the metallic single-crystal $\mathrm{Au}(111)$ substrate. Our results provide valuable insights into the fragile stability of such electronic ground states in 2D materials.

KEYWORDS: transition-metal dichalcogenide, superconductivity, charge density wave, electronic structure, angle-resolved photoemission spectroscopy, scanning tunneling microscopy, epitaxy

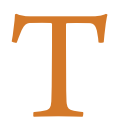
he van der Waals layered materials are an ideal playground for the investigation of exotic electronic phases in two dimensions (2D) due to greatly enhanced many-body interactions. In this regard, the metallic transitionmetal dichalcogenides (TMDs) deserve particular attention as they exhibit a rich variety of electronic phases such as charge density wave (CDW) order, ${ }^{1-5}$ superconductivity (SC), ${ }^{3,6-10}$ quantum spin liquid, ${ }^{11}$ magnetism, ${ }^{12,13}$ and, even, topological phases. ${ }^{14,15}$ Most of these electronic states rely on the strength of electron-electron and electron-phonon correlations and, therefore, are significantly sensitive to variations in the electronic and phonon band structures. Such fragility becomes critical in the $2 \mathrm{D}$ limit (single-layer, $\mathrm{SL}$ ) since the entire crystal lattice is exposed to the environment and many external factors can therefore affect their stability. Perhaps the most influential factor on the properties of $2 \mathrm{D}$ materials is the presence of the substrate, as it often triggers numerous "proximity effects" of various nature on the $2 \mathrm{D}$ material such as screening, ${ }^{16}$ charge transfer, ${ }^{17}$ hybridization, ${ }^{17-19}$ strain, ${ }^{20,21}$ etc. Although these previous works have unveiled the effect of substrates, systematic and

Received: July 15, 2021

Accepted: November 5, 2021

Published: November 30, 2021 
a)
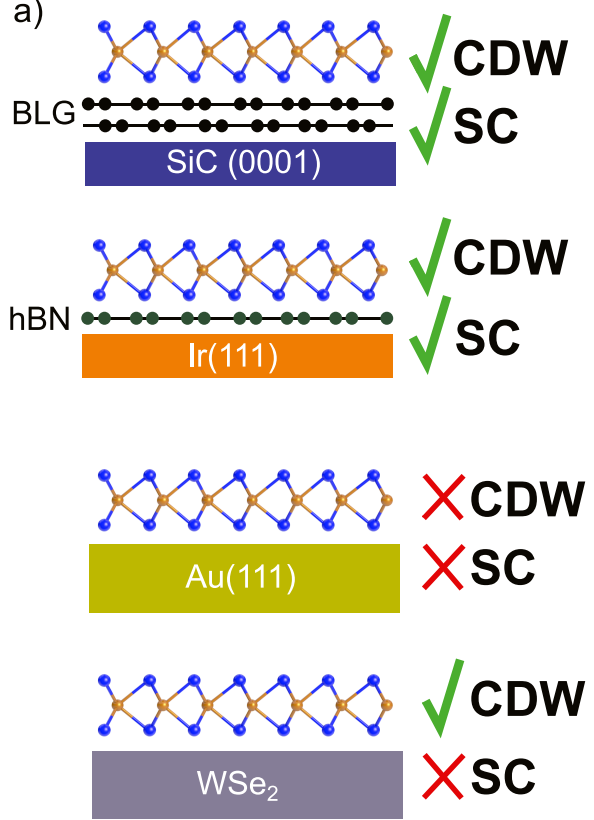

b)

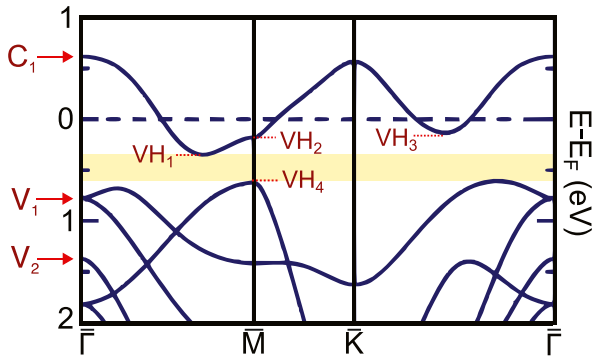

c)

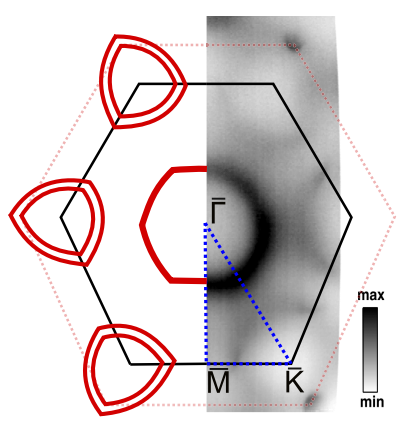

Figure 1. (a) Summary of the studied SL-NbSe 2 /substrate systems where the presence (absence) of the CDW and superconducting orders

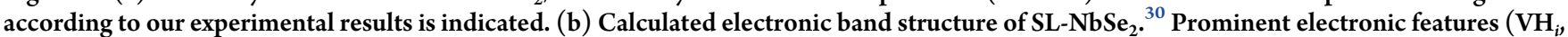
$C_{1}, V_{1}, V_{2}$ ) of the band structure are indicated. (c) Sketch of the $\mathrm{NbSe}_{2} 2 \mathrm{D} \mathrm{BZ}$ (solid hexagon) and the Fermi surface of SL-NbSe $e_{2}$ on BLG/ $\mathrm{Si}(0001)$ measured at $55 \mathrm{eV}$ photon energy. The solid red lines show the expected bands, and the high-symmetry directions are indicated by the blue dashed lines. The dashed hexagon corresponds to the 2D BZ of the BLG, and the $\bar{K}$ points for BLG can be seen in the measured data. Maximum intensity corresponds to black, and minimum intensity to white on the color scale. (b) Adapted with permission under a Creative Commons Attribution 3.0 License from ref 30. Copyright 2016 IOP Publishing.

comparative studies of their properties and impact on the collective electronic phases in $2 \mathrm{D}$ materials remain scarce.

In metallic TMDs $\left(\mathrm{MX}_{2}\right.$ with $\mathrm{M}=\mathrm{Nb}, \mathrm{Ta}, \mathrm{Ti}, \mathrm{V}, \mathrm{Sn}$ and $\mathrm{X}=\mathrm{S}$, $\mathrm{Se}, \mathrm{Te}), \mathrm{CDW}$ order and superconductivity are frequently present from the bulk form down to the single-layer limit, although with rather disparate properties in many cases. In the 2D limit, however, these electronic phases are likely influenced by the presence of the substrate; therefore, its role on the electronic structure must be carefully evaluated. So far, CDW and superconducting orders have been studied in epitaxial TMD layers on substrates that do not necessarily minimize their potential impact on the properties of the TMD layer but instead are suitable for ease and high quality growth of the TMD. These are mainly noble metals such as $\mathrm{Au}(111)^{17,22-26}$ and doped graphene surfaces, $^{2-5,9-11,27,28}$ (graphite, graphene/SiC(0001) and graphene $/ \operatorname{Ir}(111))$. In this work, we provide a comparative assessment of the impact of different substrates on the CDW and SC phases as well as the electronic structure of a model correlated 2D material. In particular, we study the electronic ground state of high-quality $\mathrm{SL}-\mathrm{NbSe}_{2}$ grown by molecular beam epitaxy (MBE) on four substrates with markedly distinct structural and electronic nature: bilayer graphene (BLG)/ $\mathrm{SiC}(0001)$, SL-boron nitride (h-BN)/Ir(111), $\mathrm{Au}(111)$, and bulk $\mathrm{WSe}_{2}$. (Details regarding the growth of SL-NbSe $\mathrm{N}_{2}$ on each substrate can be found in the Supporting Information (SI).) Surprisingly, while both CDW and SC persist with nearly identical properties on $\mathrm{BLG} / \mathrm{SiC}(0001)$ and $\mathrm{h}-\mathrm{BN} / \operatorname{Ir}(111)$, they are absent or, at least, severely weakened on $\mathrm{Au}(111)$ and bulk $\mathrm{WSe}_{2}$, as summarized in Figure 1a. However, the electronic structure of $\mathrm{SL}_{-} \mathrm{NbSe}_{2}$ remains largely unaffected in all cases except for a nonuniform shift of the band structure on $\mathrm{Au}(111)$. The fate of these collective phases is compared and discussed in the framework of the electronic structure in each case.

\section{RESULTS AND DISCUSSION}

The $2 \mathrm{H}$ polymorph of $\mathrm{NbSe}_{2}$ is a layered correlated material that hosts both CDW order and SC at low temperatures. Below $33 \mathrm{~K}$, bulk $2 \mathrm{H}-\mathrm{NbSe}_{2}$ develops a $\mathrm{CDW}$ transition showing a quasicommensurate $3 \times 3$ electronic modulation. Below $7.2 \mathrm{~K}$, this material undergoes a superconducting transition and both collective orders coexist. In the SL limit, CDW and SC have been previously detected in exfoliated layers ${ }^{7,8}$ and epitaxial layers on $\mathrm{BLG} / \mathrm{SiC}(0001) .^{3}$ The electronic band structure of undistorted $\mathrm{SL}^{-\mathrm{NbSe}_{2}}(1 \times 1)$ is dominated by a $\mathrm{Nb}$-derived $4 \mathrm{~d}$ band at the Fermi level $\left(E_{\mathrm{F}}\right)$ that sustains both the CDW and SC (see Figure 1b). ${ }^{29,30}$ This band is separated from the fully occupied Se $p_{z}$ bands, whose onset is located at $-0.8 \mathrm{eV}$, by a bandgap of $\sim 0.4$ $\mathrm{eV}$ (yellow region in Figure $1 \mathrm{~b}$ ). The band structure shows several characteristic features such as van Hove singularities $\left(\mathrm{VH}_{i}\right)$ as well as flat regions near $\bar{\Gamma}$ above $\left(C_{1}\right)$ and below $\left(V_{1}\right.$, $\left.V_{2}\right) E_{\mathrm{F}}$ that can be identified and tracked in scanning tunneling spectroscopy (STS) and angle-resolved photoemission spectroscopy (ARPES) data. ${ }^{3} \mathrm{The} \mathrm{Nb} 4 d$ states crossing the $E_{\mathrm{F}}$ form two hole pockets around the $\bar{\Gamma}$ and $\bar{K}$ points (see Figure 1c). Specifically, at the center of the 2D Brillouin zone (BZ) for SL$\mathrm{NbSe}_{2}$, marked by the solid black line, there is a hexagonal-like contour, while at the corners of the BZ there are two concentric rings corresponding to the spin-split bands at $\bar{K}$. The photoemission intensity measured at $E_{\mathrm{F}}$ was acquired with 55 $\mathrm{eV}$ photons and is shown on the right-hand side of the $\mathrm{BZ}$, while the left-hand side shows a sketch of the expected bands (we were unable to resolve the spin-split bands at $\bar{K})$. This ARPES spectrum was acquired for $\mathrm{SL}-\mathrm{NbSe}_{2}$ on $\mathrm{BLG} / \mathrm{SiC}(0001)$, whose $2 \mathrm{D} \mathrm{BZ}$ is also marked (dashed line). The point-like electronic structure of BLG near its $\bar{K}$ point is clearly visible in the shown data. 
a)

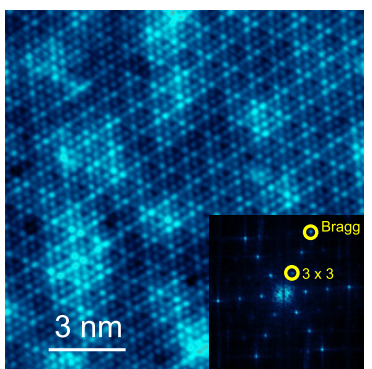

b) 1

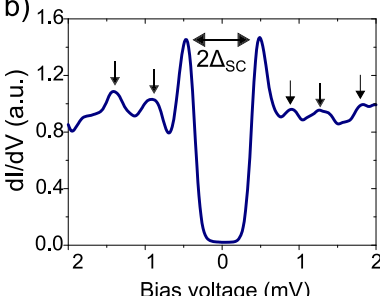

c)

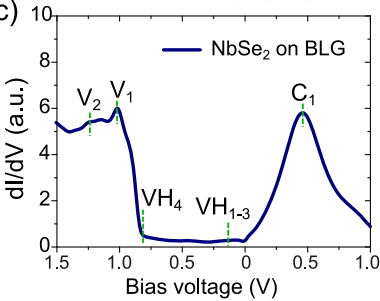

d)

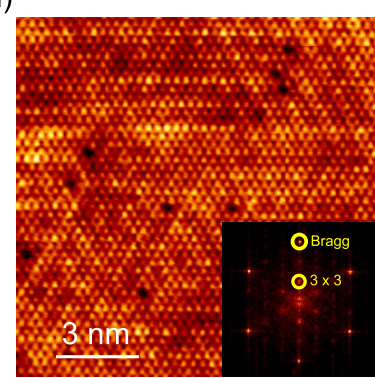

e)
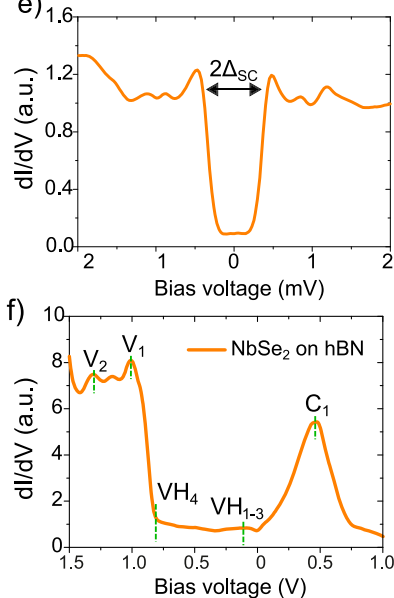

g)

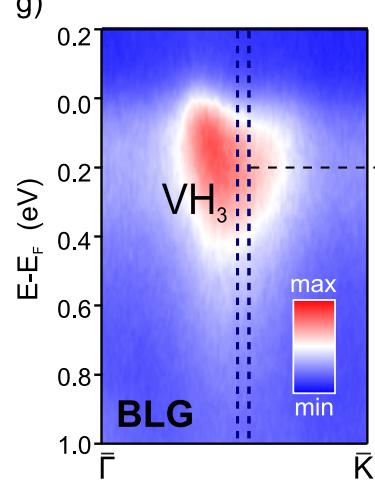

h)

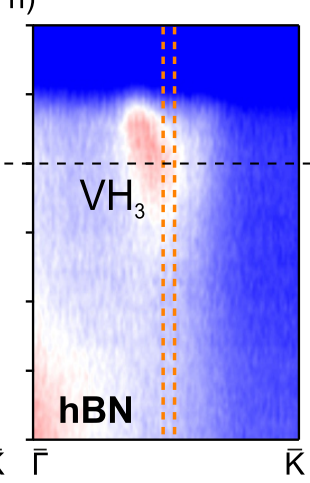

$$
\text { j) }
$$

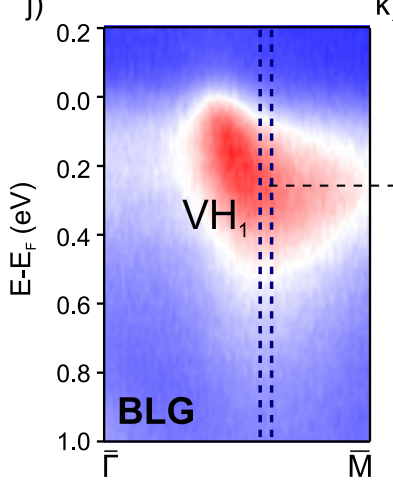

k)

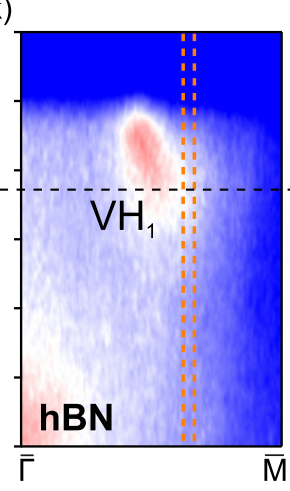

i)

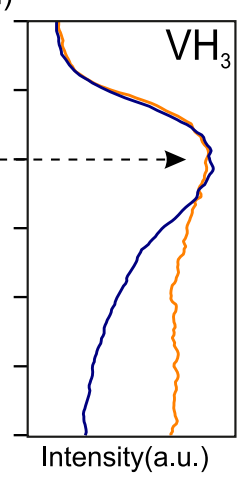

I)

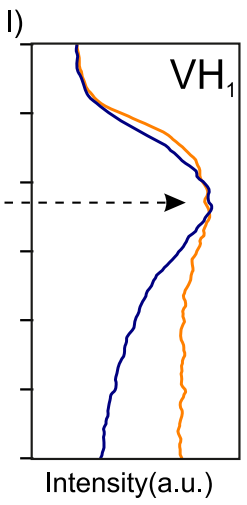

Figure 2. Structural and electronic properties of $\mathrm{SL}-\mathrm{NbSe} \mathrm{e}_{2}$ grown on $\mathrm{BLG} / \mathrm{SiC}(0001)$ and $\mathrm{h}-\mathrm{BN} / \mathrm{Ir}(111)$. (a) High-resolution $\mathrm{STM}$ topograph of SL-NbSe $e_{2}$ on $\mathrm{BLG} / \mathrm{SiC}(0001)$, where the $3 \times 3 \mathrm{CDW}$ ordering is visible at $T=340 \mathrm{mK}\left(V_{\mathrm{S}}=+50 \mathrm{mV} ; I_{\mathrm{t}}=0.86 \mathrm{nA}\right)$. The corresponding FFT is shown in the inset. (b) Low-bias and (c) high-bias STM $\mathrm{d} I / \mathrm{d} V$ spectra acquired on SL-NbSe $\mathrm{e}_{2} / \mathrm{BLG}$ at $T=340 \mathrm{mK}$. The superconducting gap $\left(\Delta_{\mathrm{SC}}\right)$, the position of collective modes (vertical arrows), and several band structure features are indicated in (b). Equivalent STM/STS measurements on $S L-N b S e_{2}$ on $\mathrm{h}-\mathrm{BN} / \mathrm{Ir}(111)$ are presented in $(\mathrm{d}-\mathrm{f})$ (parameters for $\left.(\mathrm{d}): V_{\mathrm{S}}=-50 \mathrm{mV} ; I_{\mathrm{t}}=4 \mathrm{nA}\right)$. ARPES measurements acquired with $55 \mathrm{eV}$ photons, along $\bar{\Gamma}-\bar{K}$ and $\bar{\Gamma}-\bar{M}$ high-symmetry direction of $(\mathrm{g}, \mathrm{j}) \mathrm{jL}-\mathrm{NbSe} \mathrm{e}_{2}$ on BLG and (h,k)h-BN.Dashed lines point out the band minimum. (i, l) EDCs taken at the conduction band minimum along $\bar{\Gamma}-\bar{K}$ and $\bar{\Gamma}-\bar{M}$, correspondingly. Blue color is used to represent data acquired from a SL-NbSe $e_{2} / \mathrm{BLG}$ sample, whereas orange is used for the SL-NbSe $e_{2} / \mathrm{h}-\mathrm{BN}$. The color scale used for the ARPES data is the same for all panels $(\mathrm{g}, \mathrm{h}, \mathrm{j}, \mathrm{k})$.

We first focus on the electronic structure of $\mathrm{SL}_{-} \mathrm{NbSe}_{2}$ on $\mathrm{BLG} / \mathrm{SiC}(0001)$ and $\mathrm{SL}-\mathrm{h}-\mathrm{BN} / \mathrm{Ir}(111)$. BLG/SiC (0001) is the most common substrate used to investigate the properties of $2 \mathrm{D}$ TMD metals because it is amenable to the growth process. $^{1-5,9-11,27}$ Yet, the influence of the BLG/SiC(0001) substrate on the electronic ground state of SL-TMDs remains largely unexplored. BLG is degenerately n-doped on $\mathrm{SiC}(0001)$; therefore, it behaves as a metallic substrate. To assess the influence of the metallic nature of the substrate, we quantitatively compare the properties of $\mathrm{SL}-\mathrm{NbSe}_{2}$ on BLG/ $\mathrm{SiC}(0001)$ and SL-h-BN/Ir(111), the latter a $\sim 6 \mathrm{eV}$ bandgap insulator $^{31}$ that acts as an effective decoupling layer. ${ }^{32-34}$ Figure $2 \mathrm{a}-\mathrm{c}$ and $2 \mathrm{~d}$,f summarize the scanning tunneling microscopy (STM)/STS results obtained for SL-NbSe $\mathrm{S}_{2}$ on BLG/SiC(0001) and h-BN/Ir(111), respectively. As previously reported, SL$\mathrm{NbSe}_{2}$ on BLG/SiC (0001) exhibits an unchanged $3 \times 3 \mathrm{CDW}$ with $T_{\mathrm{C}} \approx 33 \mathrm{~K}$ with respect to bulk, ${ }^{3}$ as seen in the blue colored STM topograph measured at $340 \mathrm{mK}$ (Figure 2a). Furthermore, $\mathrm{SL}_{-\mathrm{NbSe}_{2}}$ on $\mathrm{BLG} / \mathrm{SiC}(0001)$ develops superconductivity below $2 \mathrm{~K}, 3,7,8$ which is reflected in the opening of an absolute gap of $\Delta_{\mathrm{SC}}=0.40 \pm 0.02 \mathrm{meV}$ in the density of states (DOS) around $E_{\mathrm{F}}$, as seen in the $\mathrm{d} I / \mathrm{d} V$ curve of (Figure $2 \mathrm{~b}$ ) (the SC gap values $\left(\Delta_{\mathrm{SC}}\right)$ were extracted from a fit to the Dynes formula with a broadening parameter $\Gamma=10 \mu \mathrm{eV}$ ). In addition to this gap, the DOS also exhibits several peak features (vertical arrows) attributed to collective modes in the $2 \mathrm{D}$ superconductor. ${ }^{35}$
Low-temperature (340 mK) STM/STS experiments carried out on SL-NbSe ${ }_{2}$ on h-BN/Ir(111), color-coded in orange, reveal a nearly identical electronic ground state of the $\mathrm{SL}_{-} \mathrm{NbSe}_{2}$ to that on the $\mathrm{BLG} / \mathrm{SiC}(0001)$ substrate. We have confirmed that the gap feature in the SL-NbSe ${ }_{2}$ on h-BN/Ir(111) case corresponds to the superconducting gap by studying its magnetic-field dependence (see SI). High-resolution STM imaging of SL$\mathrm{NbSe}_{2}$ on h-BN/Ir(111) (Figure 2d) reveals that the CDW is fully developed on this substrate with a $3 \times 3$ periodicity. Similarly, the superconducting state is also preserved on SL$\mathrm{NbSe}_{2}$ on h-BN/Ir(111), as seen in the low-bias (i.e., $\mathrm{mV}$ range) $\mathrm{d} I / \mathrm{d} V$ spectrum shown in Figure 2e. This spectrum exhibits a superconducting gap $\left(\Delta_{\mathrm{SC}}=0.37 \pm 0.03 \mathrm{meV}\right)$ as well as the features related to collective excitations similar to the case of SL$\mathrm{NbSe}_{2}$ on BLG/SiC(0001). Unlike the BLG case, however, the DOS here shows finite conductance within the superconducting gap, which is caused by a direct tunneling channel to the supporting $\operatorname{Ir}(111)$ substrate; compare Figure $2 \mathrm{~b}$ and Figure 2e. This is consistent with previous STS experiments carried out on h-BN/Ir(111), where $\mathrm{d} I / \mathrm{d} V$ spectra contain traces of the electronic structure of the metal support. ${ }^{36}$

In order to get a detailed picture of the electronic properties of SL-NbSe ${ }_{2}$ on BLG/SiC (0001) and h-BN/Ir(111) substrates, we measured its electronic structure by STS and ARPES. Figure $2 \mathrm{c}, \mathrm{f}$ shows two typical $\mathrm{d} I / \mathrm{d} V$ spectra of $\mathrm{SL}-\mathrm{NbSe}_{2}$ taken over a largebias voltage range on $\mathrm{BLG} / \mathrm{Si}(0001)$ and $\mathrm{h}-\mathrm{BN} / \operatorname{Ir}(111)$, 
Table 1. Energies of the Main Features of the Electronic Structure As Measured by STS and ARPES ${ }^{a}$

\begin{tabular}{lcccccr}
\multicolumn{1}{c}{ substrate } & $C_{1}(\mathrm{eV})$ & $\mathrm{VH}_{1}(\mathrm{eV})$ & $\mathrm{VH}_{3}(\mathrm{eV})$ & $\mathrm{VH}_{4}(\mathrm{eV})$ & $V_{1}(\mathrm{eV})$ & $-1.30 \pm 0.07$ \\
$\mathrm{BLG} / \mathrm{SiC}$ & $0.46 \pm 0.05$ & $-0.27 \pm 0.05$ & $-0.21 \pm 0.05$ & $-0.81 \pm 0.16$ & $-1.02 \pm 0.04$ & $-1.30 \pm 0.01$ \\
$\mathrm{~h}-\mathrm{BN} / \mathrm{Ir}(111)$ & $0.43 \pm 0.06$ & $-0.29 \pm 0.05$ & $-0.23 \pm 0.05$ & $-0.80 \pm 0.02$ & $-1.03 \pm 0.03$ & - \\
$\mathrm{Au}(111)$ & $0.16 \pm 0.05$ & $-0.37 \pm 0.05$ & $-0.22 \pm 0.05$ & - & $-1.10 \pm 0.05$ & - \\
$\mathrm{WSe}_{2}$ & $0.49 \pm 0.05$ & $-0.30 \pm 0.05$ & $-0.24 \pm 0.05$ & $-0.65 \pm 0.16$ & $-0.87 \pm 0.04$ & -
\end{tabular}

${ }^{a} C_{1}, V_{1}, V_{2}$, and $\mathrm{VH}_{4}$ values were extracted from the STS data, while $\mathrm{VH}_{1}$ and $\mathrm{VH}_{3}$ were extracted from the ARPES spectra. $C_{1}, V_{1}$, and $V_{2}$ values were taken as the maxima of the STS peaks and $\mathrm{VH}_{4}$ as the onset of the Se-derived band extracted following the procedure described in ref 16. STS uncertainties were determined through a statistical analysis of individual STS curves obtained in multiple locations with different STM tips.
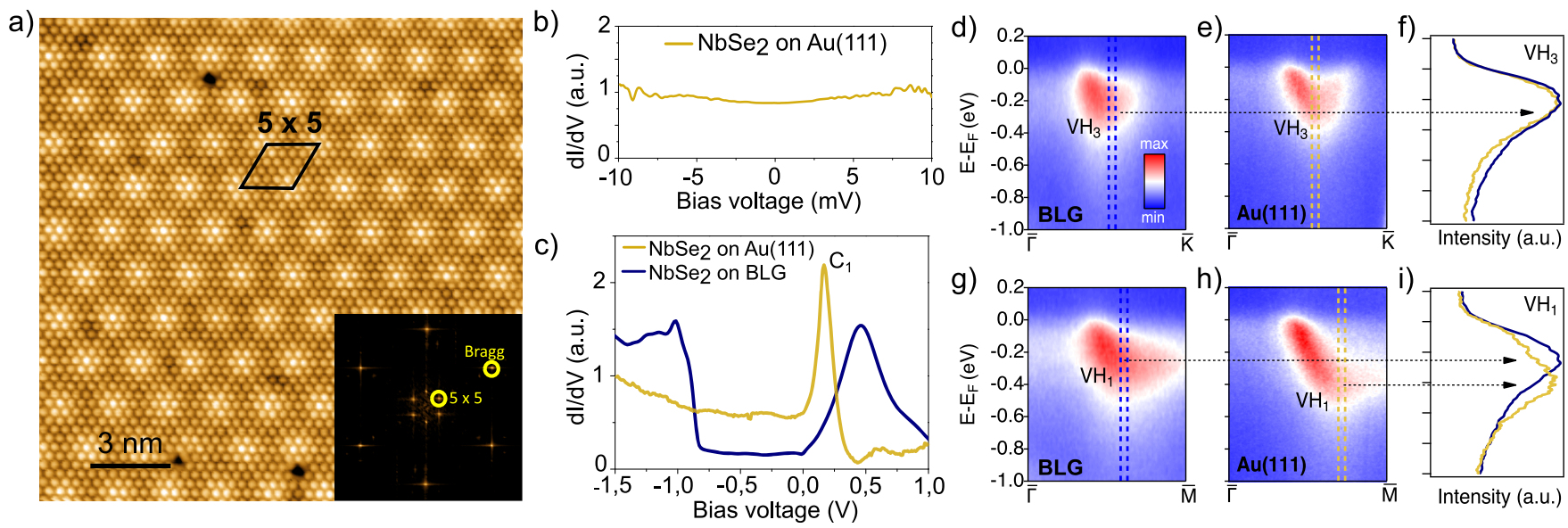

Figure 3. Structural and electronic properties of SL-NbSe $e_{2}$ on $\mathrm{Au}(111)$. (a) Atomically resolved STM image of SL-NbSe $e_{2}$ on $\mathrm{Au}(111)$ showing the $1 \times 1$ atomic lattice and a $5 \times 5$ superlattice that corresponds to a commensurate moire pattern of $17.3 \AA\left(V_{S}=+0.3 \mathrm{~V} ; I_{\mathrm{t}}=1.5 \mathrm{nA}\right)$. The corresponding FFT of the STM image is shown in the inset. (b) Low-bias and (c) large-bias STM dI/dV spectra acquired on SL-NbSe $e_{2}$ on $\mathrm{Au}(111)$ at $T=340 \mathrm{mK}$ (yellow curves). In (c), the STM dI/dV spectrum of SL-NbSe ${ }_{2}$ on BLG (blue curve) is included as a reference. ARPES measurements along $\bar{\Gamma}-\bar{K}$ and $\bar{\Gamma}-\bar{M}$ high-symmetry directions of SL-NbSe $e_{2}$ grown (d, g) on BLG and (e, h) on Au(111). Dashed lines point out the band minimum. (f, $j$ ) EDCs taken at the Nb-derived band minimum along $\bar{\Gamma}-\bar{K}$ and $\bar{\Gamma}-\bar{M}$, correspondingly. The color scale is the same for all panels $(d, e, g, h)$.

respectively. The observed electronic structure is nearly identical in both cases and fairly coincident with several band structure features sketched in Figure $1 \mathrm{~b}$ : (a) a pronounced peak at sample bias $V_{\mathrm{S}}=+0.43 \mathrm{~V}$ (empty states) that corresponds to the flat region at the top of the $\mathrm{Nb}$-derived band at $\bar{\Gamma}\left(C_{1}\right.$ in Figure $1 \mathrm{~b})$; (b) a shallow peak near $V_{\mathrm{S}}=-0.20 \mathrm{~V}$ contributed by the van Hove singularities below $E_{\mathrm{F}}\left(\mathrm{VH}_{1}, \mathrm{VH}_{2}\right.$, and $\left.\mathrm{VH}_{3}\right)$ (see $\mathrm{SI})$; (c) a region of low and flat DOS attributed to the bandgap of $0.4 \mathrm{eV}$; (d) a sharp increase of the DOS whose onset is at $V_{\mathrm{S}}=$ $-0.82 \mathrm{~V}$ and is consistent with the top of the Se-derived band at the $\bar{M}$-point $\left(\mathrm{VH}_{4}\right)$ according to recent theory reports; ${ }^{29,30}$ and (e) two peaks at $V_{\mathrm{S}}=-1.03 \mathrm{~V}$ and $V_{\mathrm{S}}=-1.30 \mathrm{~V}$ that can be attributed to the $V_{1}$ and $V_{2}$ features in the band structure. All these STS features are coincident in energy in the two systems within uncertainties (see Table 1).

To further clarify the electronic structure in these two systems, we performed ARPES measurements at room temperature (RT). The band dispersion along the $\bar{\Gamma}-\bar{K}$ and $\bar{\Gamma}-\bar{M}$ highsymmetry directions, indicated by the blue dashed lines in Figure 1c, are respectively presented for the cases of SL-NbSe on $\mathrm{BLG} / \mathrm{SiC}(0001)$ and $\mathrm{h}-\mathrm{BN} / \mathrm{Ir}(111)$ in Figure $2 \mathrm{~g}, \mathrm{j}$ and $2 \mathrm{~h}, \mathrm{k}$. The position of the band minimum was determined by fitting energy distribution curves (EDCs). The band minimum in the $\bar{\Gamma}-\bar{K}$ direction $\left(\mathrm{VH}_{3}\right)$ for $\mathrm{SL}-\mathrm{NbSe}_{2}$ on $\mathrm{BLG} / \mathrm{SiC}(0001)$ is at $\sim 210 \mathrm{meV}$, while it is $\sim 230 \mathrm{meV}$ for the case of SL-NbSe ${ }_{2}$ on h$\mathrm{BN} / \mathrm{Ir}(111)$. A similar shift of the $\mathrm{Nb} 4 \mathrm{~d}$ band to lower binding energy is observed in the $\bar{\Gamma}-\bar{M}$ direction $\left(\mathrm{VH}_{1}\right)$, as shown in the EDCs of Figure 2i,l. We do not observe any detectable hybridization (i.e., avoided crossing) between the SL-NbSe bands and the h-BN and BLG bands, which indicates a weak interaction between the $\mathrm{SL}-\mathrm{NbSe}_{2}$ and the substrate; however given the resolution of our acquired data, we cannot fully rule out this possibility. In light of the STS and ARPES comparative measurements, we conclude that the electronic structure remains unaltered by replacing $\mathrm{BLG} / \mathrm{SiC}(0001)$ with h-BN/ $\operatorname{Ir}(111)$ as the substrate, which is consistent with the observation of CDW order and SC in both cases. The metallicity of the graphene (i.e., finite DOS at $E_{\mathrm{F}}$ ) does not noticeably impact the superconducting state of the $\mathrm{SL}-\mathrm{NbSe}_{2}$. This is relevant as graphene is a common substrate for the growth and further investigation of TMD metals. ${ }^{1-5,9-11,27}$ In the following, we will use the $\mathrm{SL}-\mathrm{NbSe}_{2}$ on BLG/SiC(0001) system as a reference, as it represents to good approximation the freestanding electronic structure of SL-NbSe ${ }_{2}$.

Next, we focus on the electronic structure of SL-NbSe ${ }_{2}$ on $\mathrm{Au}(111)$ following the same procedure. Figure $3 \mathrm{a}$ shows an atomically resolved STM image of $\mathrm{SL}^{-\mathrm{NbSe}_{2}}$ on the $\mathrm{Au}(111)$ surface, where, in addition to the atomic registry, a rotationally aligned superstructure is clearly observed. This superlattice has a commensurate $5 \times 5$ periodicity with respect to $\mathrm{SL}-\mathrm{NbSe}_{2}$ whose origin is a moire pattern formed between the TMD and the Au atomic lattice. ${ }^{23}$ Fast Fourier transform (FFT) analysis of STM topographs (inset in Figure $3 a$ ) shows that the $5 \times 5$ superlattice is the only existing periodicity in $\mathrm{SL}_{-} \mathrm{NbSe}_{2}$ on $\mathrm{Au}(111)$ beyond the atomic lattice; therefore, there is no trace of $3 \times 3 \mathrm{CDW}$ ordering. The low-energy electronic structure near $E_{\mathrm{F}}$ probed by STS at $340 \mathrm{mK}$ in Figure $3 \mathrm{~b}$ shows a flat DOS, 
a)

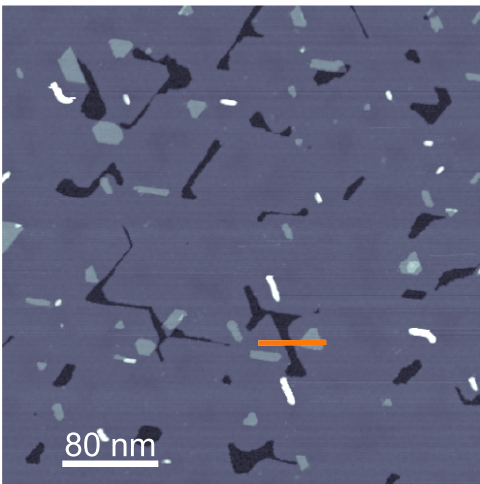

b)

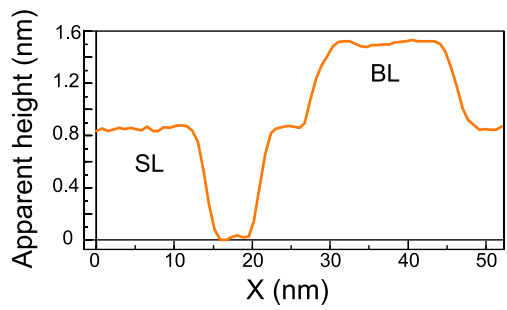

c)

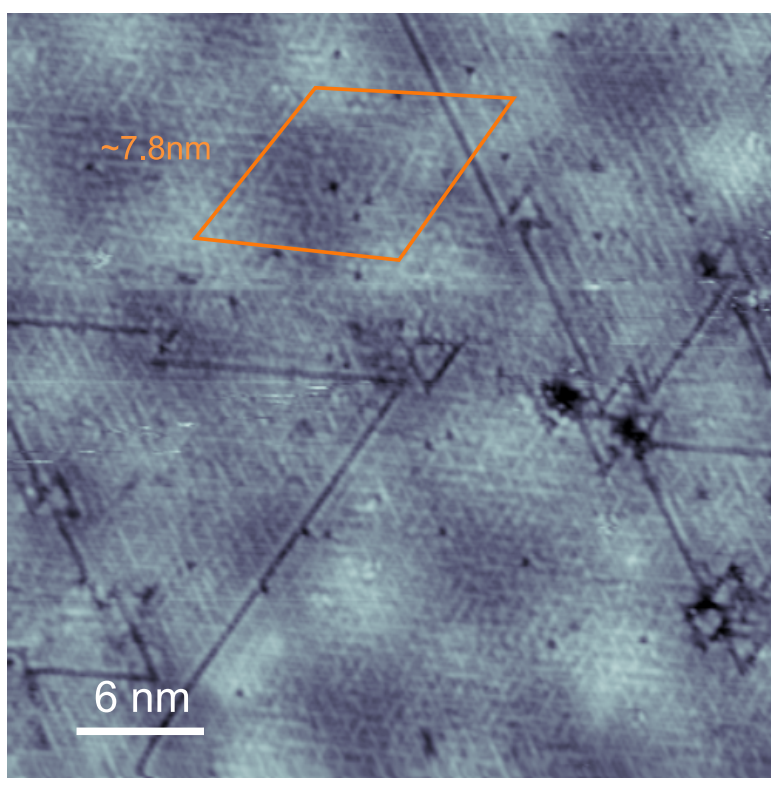

Figure 4. Morphology of SL-NbSe ${ }_{2}$ on bulk WSe $e_{2}$ (a) Large-scale STM image of a nearly complete monolayer of $\mathrm{NbSe}_{2}$ grown on bulk WSe $_{2}\left(V_{\mathrm{S}}\right.$ $=+0.93 \mathrm{~V} ; I_{\mathrm{t}}=30 \mathrm{pA}, T=4.2 \mathrm{~K}$ ). (b) Height profile taken along the orange line in (a) that illustrates the apparent heights of the monolayer and the bilayer. (c) Close-up STM image of SL-NbSe ${ }_{2} / \mathrm{WSe}_{2}$ showing a moire superlattice with a length of $\sim 7.8 \mathrm{~nm}$ arising from the lattice mismatch between $S L-\mathrm{NbSe}_{2}(3.44 \AA)$ and $\mathrm{WSe}_{2}(3.29 \AA)$ at zero angle between them $\left.\left(V_{S}=+1.8 \mathrm{~V} ; I_{\mathrm{t}}=3 \mathrm{nA} ; T=4.2 \mathrm{~K}\right) .4 .2 \mathrm{~K}\right)$.
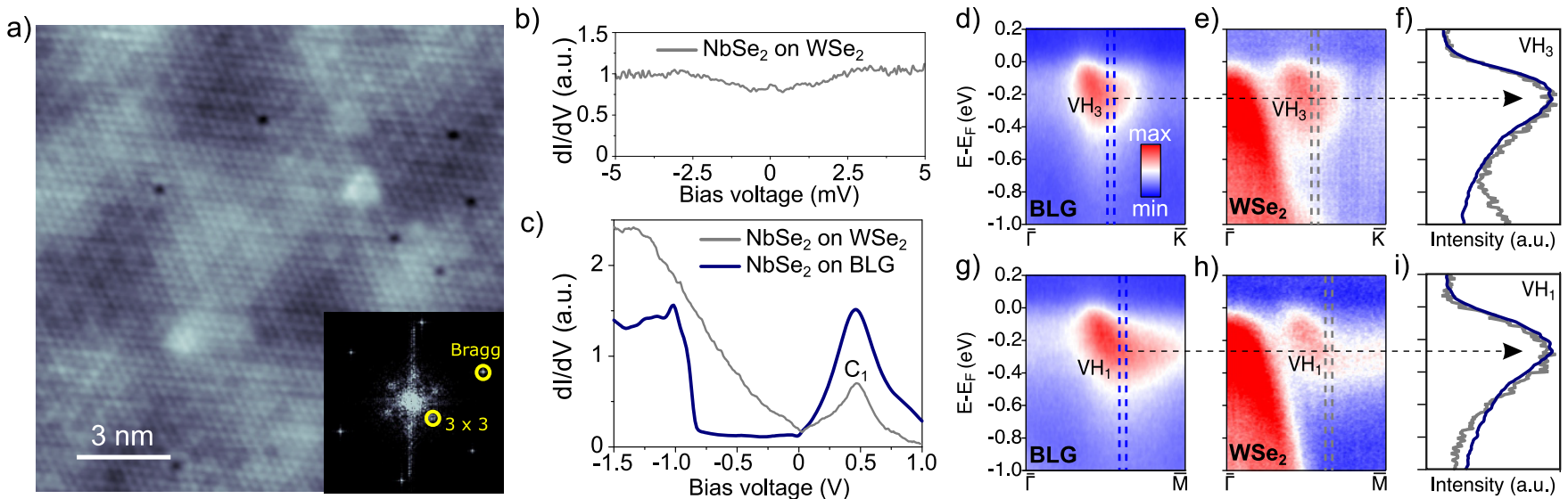

Figure 5. Structural and electronic properties of SL-NbSe ${ }_{2}$ grown on bulk WSe $e_{2}$ (a) Atomically resolved STM image showing a barely visible $3 \times$ $3 \mathrm{CDW}$ modulation that leads to a blurred $3 \times 3$ points in the FFT (this has been observed systematically with different tip apexes, see SI $)\left(V_{\mathrm{S}}=\right.$ $-0.5 \mathrm{~V} ; I_{\mathrm{t}}=0.15 \mathrm{nA}$ ). (b) Low-bias STM dI/dV spectrum showing the absence of the SC gap but a shallow dip feature attributed to the CDW order. (c) Wide-range STM $\mathrm{d} I / \mathrm{d} V$ spectrum where only $C_{1}$ is distinguished. ARPES measurements along $\bar{\Gamma}-\bar{K}$ and $\bar{\Gamma}-\bar{M}$ high-symmetry directions of $\mathrm{SL} \mathrm{NbSe}_{2}$ grown on $(\mathrm{d}, \mathrm{g}) \mathrm{BLG} / \mathrm{SiC}(0001)$ and $(\mathrm{e}, \mathrm{h})$ bulk WSe $\mathrm{H}_{2}$. The black dashed lines point to the band minimum in $(\mathrm{f}, \mathrm{i})$ where EDCs acquired at the conduction band minimum along the corresponding $\bar{\Gamma}-\bar{K}$ and $\bar{\Gamma}-\bar{M}$ directions. Comparison of the $V_{1}, V_{2}$, and $V_{H}$ features for $\mathrm{SL}-\mathrm{NbSe}_{2}$ on $\mathrm{BLG} / \mathrm{SiC}(0001)$, and bulk WSe $\mathrm{W}_{2}$; they all appear to similar position in energy. The color scale is the same for all panels $(\mathrm{d}, \mathrm{e}, \mathrm{g}, \mathrm{h})$.

which demonstrates the quenching of SC by the proximity of the normal metal.

Figure $3 \mathrm{c}$ shows two typical wide-bias $\mathrm{d} I / \mathrm{d} V$ spectra measured for $\mathrm{SL}_{-\mathrm{NbSe}}$ on $\mathrm{Au}(111$ ) (yellow) and, for comparison, on $\mathrm{BLG} / \mathrm{SiC}(0001)$. For occupied states, the DOS of SL-NbSe $e_{2}$ on $\mathrm{Au}(111)$ shows a featureless monotonic increase of the DOS that we attribute to the dominant weight of the direct tunnelling channel to the bulk bands of $\mathrm{Au}(111)$. Importantly, we do not observe any STS feature compatible with the surface state of $\mathrm{Au}(111)$. Lastly, although the $V_{1}$ and $V_{2}$ features from the Se $p_{z}$ bands are not observed in STS in contrast to the BLG case, the dispersion and position of the former is detected in our ARPES measurements and found to be nearly unaltered with respect to the BLG system (see Table 1 and SI). For unoccupied states, we find $C_{1}$ for $\mathrm{SL}_{-} \mathrm{NbSe}_{2}$ on $\mathrm{Au}(111)$ to be located at $V_{\mathrm{S}}=+0.16 \mathrm{~V}$. This value represents a surprisingly large shift of $C_{1}$ of $300 \mathrm{meV}$ toward $E_{\mathrm{F}}$ with respect to the SL$\mathrm{NbSe}_{2} / \mathrm{BLG}$ system. This shift along with the simultaneous disappearance of the $\mathrm{Au}(111)$ surface state suggests charge transfer from the substrate to the $2 \mathrm{D}$ layer. This scenario is consistent with the ARPES data shown in Figure 3d-i, where the $\mathrm{Nb} 4 d$ band moves further below $E_{\mathrm{F}}$ compared to the weakly interacting SL-NbSe 2 /BLG case. However, this band does not show a rigid shift as it would be expected for a bare charge transfer. While the band position in the $\bar{\Gamma}-\bar{K}$ direction $\left(\mathrm{VH}_{3}\right)$ for $\mathrm{Au}(111)$ and BLG does not reveal any qualitative difference, 
there is a clear shift of the band in the $\bar{\Gamma}-\bar{M}$ direction $\left(\mathrm{VH}_{1}\right)$ of $\sim 100 \mathrm{meV}$. Regarding the $\mathrm{Au}(111)$ surface state, our ARPES data cannot resolve it due to the nearly full coverage of SL-NbSe (partial TMD coverages enable its observation).

The disappearance of the $\mathrm{Au}(111)$ surface state, the large shift of the $C_{1}$ feature toward $E_{\mathrm{F}}$, and the nonuniform shift of the $\mathrm{Nb}$ derived band are fully compatible with pseudodoping effects similar to those observed in $\mathrm{SL}-\mathrm{TaS}_{2} / \mathrm{Au}(111)^{38}$ and arising from charge transfer and, more importantly, hybridization between the d-band of the TMD and the $\mathrm{Au}(111)$ surface state. First-principles calculations have shown for SL-TaS ${ }_{2}$ that the $d$ band can shift unequally in energy in different points in $k$ space. ${ }^{17}$ Since the pseudodoping-induced shifts of the bands can be $k$-dependent within the $\mathrm{BZ}$, the states above and below $E_{\mathrm{F}}$ can be affected by different amounts, which is consistent with the measured larger shift of the unoccupied $C_{1}$ STS feature $(\sim 300$ $\mathrm{meV})$ than that detected in the occupied region by ARPES $(\sim$ $100 \mathrm{meV}$ ) as seen in Figure 3c,i. Therefore, the hybridization of the $\mathrm{Nb} 4 d$ band with the Au surface state leads to nonuniform ( $k$-dependent) band shifts that reshape the electronic structure of the 2D layer. Pseudodoping impacts the CDW and superconducting states in $\mathrm{SL}-\mathrm{NbSe}_{2}$ likely through a reduction of the DOS as a result of the hybridization that leads to a decrease of the effective coupling constants upon deposition on $\mathrm{Au}(111)$, which contribute toward their disappearance. In the case of the SC, the Cooper pair leakage to the Au(111) substrate due to boundary effects is also expected to play a large role in its quenching. $^{39}$

Lastly, we investigate the case of SL- $\mathrm{NbSe}_{2}$ on a large-gap (>1 $\mathrm{eV}$ ) semiconductor, that is, bulk WSe $\mathrm{e}_{2}$. The morphology of SL$\mathrm{NbSe}_{2}$ grown on $\mathrm{WSe}_{2}$ is shown in Figure $4 \mathrm{a}$. The high quality of the crystal structure is revealed by the uniform shape of the layer and straight edges of the domains. The $\mathrm{SL}-\mathrm{NbSe}_{2}$ grows with both atomic lattices rotationally aligned. This alignment is confirmed by constant energy maps acquired by ARPES (not shown). However, due to the lattice mismatch between SL$\mathrm{NbSe}_{2}(3.44 \AA)$ and $\mathrm{WSe}_{2}(3.29 \AA)$, a large moiré pattern with a periodicity of $7.8 \mathrm{~nm}$ is formed in the heterostructure, which is clearly visible in STM images (Figure 4c). Regarding the existence of CDW and SC, we proceed as we did with previous substrates. High-resolution STM imaging of $\mathrm{SL}_{-}-\mathrm{NbSe}_{2}$ on bulk $\mathrm{WSe}_{2}$ (Figure 5a) reveals a superlattice compatible with the existence of CDW order with a ratio between the Bragg $\left(b_{1 \times 1}\right)$ and superlattice $\left(b_{\mathrm{SL}}\right)$ reciprocal vectors of $b_{1 \times 1} / b_{\mathrm{SL}}=3.10 \pm$ 0.53 . However, the spots of the superlattice in the corresponding FFT (inset) are rather blurred and poorly defined. This situation, and even the absence of CDW, is systematically observed in our STM images acquired in this system (see SI), which indicates an intrinsic weakening of the CDW on bulk $\mathrm{WSe}_{2}$ as compared to the BLG and h-BN cases. Concerning the superconducting state, the DOS near $E_{\mathrm{F}}$ usually exhibits a Vshape (see SI), whose relation with the SC state has to be considered. To do so, we consecutively acquired STM $\mathrm{d} I / \mathrm{d} V$ spectra of SL- $\mathrm{NbSe}_{2}$ on bulk WSe $\mathrm{S}_{2}$ below $(0 \mathrm{~T})$ and above $(5 \mathrm{~T})$ the upper critical magnetic field $(2.2 \mathrm{~T}$ ) at $340 \mathrm{mK}$ (see SI), which show a nearly identical shape. The normalized $\mathrm{d} I / \mathrm{d} V$ spectrum $(\mathrm{d} I / \mathrm{d} V(0 \mathrm{~T}) / \mathrm{d} I / \mathrm{d} V(5 \mathrm{~T}))$ is shown in Figure $5 \mathrm{~b}$. The normalized DOS shows a shallow dip of $\sim 6 \mathrm{meV}$ that can only be related to the CDW gap. Therefore, we rule out the presence of SC in SL-NbSe $e_{2}$ on the bulk WSe $e_{2}$ substrate.

To gain knowledge about the weakening of the CDW and the disappearance of $\mathrm{SC}$ in $\mathrm{SL}-\mathrm{NbSe}_{2}$ grown on the semiconducting bulk $\mathrm{WSe}_{2}$ substrate, we study its electronic structure via STS and ARPES. Figure $5 \mathrm{c}$ shows a typical $\mathrm{d} I / \mathrm{d} V$ spectrum of this system along with the $\mathrm{SL}-\mathrm{NbSe}_{2} / \mathrm{BLG}$ reference spectrum. For unoccupied states, the only feature is the broad $C_{1}$ peak centered at $V_{\mathrm{S}}=+0.49 \mathrm{eV}$, nearly coincident in energy with that for SL$\mathrm{NbSe}_{2}$ on $\mathrm{BLG} / \mathrm{SiC}(0001)$. For occupied states, the DOS shows a continuous and large increase from $E_{\mathrm{F}}$ that dominates over other STS features such as $\mathrm{VH}_{i}$ or $V_{1,2}$, thus rendering them not visible. We attribute the large increase of DOS to tunneling from the bulk valence bands of $\mathrm{WSe}_{2}$, whose onset in $\mathrm{SL}-\mathrm{NbSe}_{2}$ on $\mathrm{WSe}_{2}$ is located very close to $E_{\mathrm{F}}$ (see ARPES spectra in Figure $5 e, h)$. The bulk WSe $e_{2}$ valence band maximum is located at the $\bar{\Gamma}$ point and has high intensity (red) with noticeable dispersion in the $k_{z}$ direction, ${ }^{40}$ which maximizes the tunneling probability to these states and, therefore, their weight in the measured $\mathrm{d} I / \mathrm{d} V$ spectra. Figure $5 \mathrm{~d}$,e and $5 \mathrm{~g}, \mathrm{~h}$ compares the dispersion of the SL$\mathrm{NbSe}_{2}$ states on BLG and bulk WSe $e_{2}$ along the $\bar{\Gamma}-\bar{K}$ and $\bar{\Gamma}-\bar{M}$ directions, respectively. ARPES data do not reveal a significant shift of the band minimum in the $\bar{\Gamma}-\bar{K}$ high-symmetry direction as compared to the reference spectra for $\mathrm{SL}-\mathrm{NbSe}_{2}$ on BLG/ $\mathrm{SiC}(0001)$ and h-BN/Ir(111) substrates. The shift of the pocket in the $\bar{\Gamma}-\bar{M}$ direction is equal with the $\mathrm{SL}-\mathrm{NbSe}_{2}$ on BLG/ $\mathrm{SiC}(0001)$ and $\mathrm{h}-\mathrm{BN} / \operatorname{Ir}(111)$ samples (compare EDCs in Figures 21 and $5 i$ ). In summary, from the STS and ARPES data, we conclude that the electronic structure of SL-NbSe ${ }_{2}$ remains unaffected within our experimental resolution by the presence of the bulk $\mathrm{WSe}_{2}$ substrate with respect to the reference substrates of $\mathrm{BLG} / \mathrm{SiC}(0001)$ and h-BN/Ir(111) .

This result allows us to draw some conclusions about the behavior of CDW and SC in this system. First, the nearly equal electronic structure of $\mathrm{SL}_{-} \mathrm{NbSe}_{2}$ on bulk $\mathrm{WSe}_{2}, \mathrm{BLG} /$ $\mathrm{SiC}(0001)$, and $\mathrm{h}-\mathrm{BN} / \mathrm{Ir}(111)$ substrates rules out significant charge transfers and/or changes in the Fermi surface that could critically affect the stability of these collective states. Similarly, hybridization between $\mathrm{Nb} 4 d$ states and substrate states can also be discarded due to the absence of overlap. Furthermore, in none of the cases we observe the emergence of defect states due to a significantly high density of defects, which would be detrimental to the SC phase but not necessarily the CDW phase, as we have observed $\mathrm{CDW}$ and SC in heavily doped SL$\mathrm{Nb}_{1-\delta} \mathrm{Mo}_{\delta} \mathrm{Se}_{2}$ alloys for large Mo concentrations up to $15 \%{ }^{41}$

Weakening of the CDW order and complete suppression of $\mathrm{SC}$ for SL-NbSe ${ }_{2}$ could be due to minute but measurable energy shifts of the van Hove singularities. For bulk $2 \mathrm{H}-\mathrm{NbSe}_{2}$, the energy difference between the band minima along the $\Gamma-K$ and $\Gamma-M$ directions is $\sim 0.1 \mathrm{eV}$. In the SL case, comparison of the EDCs displayed in Figure 5i reveals a plausible smaller energy difference and, therefore, a closer position of $\mathrm{VH}_{1}$ to $E_{\mathrm{F}}$ with respect to the bulk case. Similar shifts with strong impact in the CDW and superconducting orders have been previously reported in bulk TMDs. ${ }^{42}$ Furthermore, the presence of longwavelength moire pattern $(7.8 \mathrm{~nm})$ in $\mathrm{SL}-\mathrm{NbSe}_{2}$ on bulk $\mathrm{WSe}_{2}$ is a distinctive feature with respect to the reference substrates (where both CDW and SC are seen) that could be affecting the stability of the CDW and SC. Although long moire patterns are present in all the heterostructures studied here, the induced structural/electronic corrugation in the $\mathrm{NbSe}_{2}$ layer is only noticeable via STM on the bulk WSe ${ }_{2}$ substrate. Further work to assess the potential impact of moirés in $2 \mathrm{D}$ TMD superconductors as well as other plausible factors is required to reveal the microscopic mechanisms that lead to the weakening and disappearance of the CDW and SC, respectively, in SL-NbSe ${ }_{2}$ on bulk WSe . . 


\section{CONCLUSION}

In conclusion, high-resolution STM/STS and ARPES measurements allowed us to interrogate the impact of the substrate on the electronic structure and collective ground states (CDW and $\mathrm{SC}$ ) in SL-NbSe 2 , a model correlated 2D material. A key insight gained in this study is the significance of the fact that the BLG/ $\mathrm{SiC}(0001)$ substrate plays a negligible role in the electronic properties of the $2 \mathrm{D}$ TMD metal, as demonstrated by direct comparison with SL-NbSe ${ }_{2}$ on h-BN/Ir(111). Our measurements also reveal a nearly identical electronic structure of SL$\mathrm{NbSe}_{2}$ on BLG/SiC(0001), h-BN/Ir(111), and bulk $\mathrm{WSe}_{2}$ substrates, which can be considered close to the free-standing case. However, while both $\mathrm{CDW}$ and $\mathrm{SC}$ in $\mathrm{SL}-\mathrm{NbSe}_{2}$ are present on $\mathrm{BLG} / \mathrm{SiC}(0001)$ and $\mathrm{h}-\mathrm{BN} / \mathrm{Ir}(111)$, they are both critically impacted in the case of bulk WSe $\mathrm{W}_{2}$. Lastly, the $\mathrm{Au}(111)$ substrate is not suitable for fundamental studies of the intrinsic electronic structure of $2 \mathrm{D}$ TMD metals. Our study presents a model case with valuable insights that we expect will contribute toward a better understanding of relevant substrate-induced proximity effects in $2 \mathrm{D}$ materials.

\section{METHODS}

In this section, we outline the preparation of the substrates themselves as well as the subsequent growth of SL-NbSe $e_{2}$ on each of them. Details regarding the STM/STS and ARPES measurements are also provided.

Substrates Preparation. Bilayer Graphene. BLG was epitaxially grown on $\operatorname{SiC}(0001)$ wafers with resistivities $\rho \approx 120 \Omega \cdot \mathrm{cm}$. First, the $\mathrm{SiC}(0001)$ wafers (previously rinsed with an isopropyl solution) were inserted into an UHV-MBE chamber (base pressure of $5 \times 10^{-10} \mathrm{mbar}$ ) and outgassed at $650{ }^{\circ} \mathrm{C}$ for $30 \mathrm{~min}$. Next, the BLG was grown by heating the sample to $1400{ }^{\circ} \mathrm{C}$ for another $35 \mathrm{~min}$. During the growth, we monitored the emergence and quality of the BLG via reflection highenergy electron diffraction (RHEED).

Bulk $\mathrm{WSe}_{2}$. WSe $\mathrm{W}_{2}$ crystals were exfoliated in air and immediately introduced into the UHV-MBE system. The bulk WSe $\mathrm{W}_{2}$ substrates were subsequently outgassed at $650{ }^{\circ} \mathrm{C}$ for $10 \mathrm{~min}$ prior to the SL-NbSe $\mathrm{N}_{2}$ growth.

$A u(111) . A u(111)$ single crystals were cleaned by carrying out multiple cycles of $\mathrm{Ar}^{+}$sputtering $(V=0.5 \mathrm{keV})$ and annealing $(T=550$ $\left.{ }^{\circ} \mathrm{C}\right)$ in the UHV-MBE chamber.

$S L-h-B N$ on $\operatorname{Ir}(111)$. SL-h-BN on a $\operatorname{Ir}(111)$ surface was prepared in an UHV system (base pressure: $<3 \times 10^{-10} \mathrm{mbar}$ ) that is equipped with a variable-temperature (VT)-STM and standard surface preparation facilities. The single crystal $\operatorname{Ir}(111)$ was cleaned by $\mathrm{Ar}^{+}$sputtering while maintaining the substrate at $850{ }^{\circ} \mathrm{C}$ for $60 \mathrm{~min}$, followed by annealing at $850^{\circ} \mathrm{C}$ in an oxygen atmosphere at a partial pressure of $2.7 \times 10^{-6} \mathrm{mbar}$ during $40 \mathrm{~min}$ and finishing with a short annealing at $900{ }^{\circ} \mathrm{C}$ and subsequent flash-anneal at $1050^{\circ} \mathrm{C}$ under UHV conditions. SL-h-BN was grown via chemical vapor deposition employing borazine $\left(\mathrm{B}_{3} \mathrm{H}_{6} \mathrm{~N}_{3}\right)$ as the precursor molecule. Specifically, the as-cleaned $\operatorname{Ir}(111)$ surface, kept at $950{ }^{\circ} \mathrm{C}$, was exposed to 75 Langmuir of borazine at a partial pressure of $3.3 \times 10^{-8} \mathrm{mbar}$. This procedure yields fully covered, well-aligned, and single domain of SL-h-BN on $\operatorname{Ir}(111)$ (see SI). Once the surface quality and cleanliness were examined by STM at RT, the sample was air-transferred to the MBE system for the growth of SL-NbSe $e_{2}$. To avoid degradation due to air exposure during transfer between systems, the h-BN/Ir(111) surface was coated with 25-6 ML of PTCDA molecules as a capping layer. In the MBE chamber, the PTCDA molecules were thermally desorbed from the substrate by annealing at $850{ }^{\circ} \mathrm{C}$ for $30 \mathrm{~min}$ before the growth of SL$\mathrm{NbSe}_{2}$.

Epitaxial Growth of SL-NbSe $e_{2}$. Single layers of $\mathrm{NbSe}_{2}$ were grown by MBE on epitaxial BLG on $6 \mathrm{H} \operatorname{SiC}(0001)$, h-BN on $\operatorname{Ir}(111)$, $\mathrm{Au}(111)$, and bulk WSe ${ }_{2}$. The base pressure of the MBE chamber was 5 $\times 10^{-10}$ mbar. High-purity $\mathrm{Nb}(99.99 \%)$ and Se (99.999\%) were evaporated from an e-beam evaporator and a standard Knudsen cell, respectively. The $\mathrm{Nb}$ :Se flux rate was kept at 1:30 for all the substrates.
The table below shows the optimized growth parameters (substrate temperature and time) to obtain a $\mathrm{NbSe}_{2}$ coverage of $\sim 0.9 \mathrm{ML}$ for each substrate. The growth of the $\mathrm{NbSe}_{2}$ was monitored by in situ RHEED measurements. Atomic force microscopy at ambient conditions was routinely used to optimize the morphology of the $\mathrm{NbSe}_{2}$ layers. In order to transfer the samples from our UHV-MBE system to the STM (San Sebastián, Spain) or ARPES (Aarhus, Denmark), the films were capped with a $\sim 10 \mathrm{~nm}$ film of Se to protect them against oxidation and moisture. The Se-capping layer was easily removed under UHV conditions by annealing the sample to $\sim 280{ }^{\circ} \mathrm{C}$ (Table 2).

Table 2. Optimized Growth Parameters of Single-Layer $\mathrm{NbSe}_{2}$ on Various Substrates

\begin{tabular}{lcc}
\multicolumn{1}{c}{ substrate } & temperature $\left({ }^{\circ} \mathrm{C}\right)$ & growth time $(\mathrm{min})$ \\
$\mathrm{BLG} / \mathrm{SiC}(0001)$ & 580 & 30 \\
$\mathrm{WSe}$ & 550 & 25 \\
$\mathrm{Au}(111)$ & 560 & 20 \\
$\mathrm{~h}-\mathrm{BN} / \mathrm{Ir}(111)$ & 590 & 45 \\
\hline
\end{tabular}

STM/STS Measurements. STM/STS experiments were carried out in a commercial UHV, low-temperature, and high-magnetic field STM USM-1300 from Unisoku operating at $T=0.34 \mathrm{~K}$ unless otherwise stated. STS measurements (using Pt/Ir tips) were performed using the lock-in technique with typical a.c. modulations of $20-50 \mu \mathrm{V}$ at $833 \mathrm{~Hz}$ for the low-bias spectra and $2-6 \mathrm{mV}$ at $833 \mathrm{~Hz}$ for the largebias spectra. STM/STS data were analyzed and rendered using WSxM software. $^{43}$ To avoid tip artifacts in our STS measurements, the STM tips we used for our experiments were previously calibrated using a $\mathrm{Cu}(111)$ surface as reference. A tip was considered calibrated only when STS performed on $\mathrm{Cu}(111)$ showed a sharp surface state onset at $-0.44 \mathrm{eV}$ followed by a clean, monotonic decay of the $\mathrm{d} I / \mathrm{d} V$ signal. $^{35}$ In addition to this, we also inspected the DOS within $\pm 10 \mathrm{mV}$ to avoid the inadvertent use of functionalized tips with strong variations in the DOS around $E_{\mathrm{F}}$.

ARPES Measurements. ARPES data were taken using $p$-polarized light at a photon energy of $55 \mathrm{eV}$ at the SGM3 beamline of the ASTRID2 synchrotron light source in Aarhus, Denmark. ${ }^{44}$ Energy and angular resolution were $\approx 40 \mathrm{meV}$ and $0.2^{\circ}$, respectively. The sample was fixed at room temperature during the measurements, which is above $T_{\mathrm{CDW}}$ and $T_{\mathrm{C}}$.

\section{ASSOCIATED CONTENT}

\section{(I) Supporting Information}

The Supporting Information is available free of charge at https://pubs.acs.org/doi/10.1021/acsnano.1c06012.

The structural properties of the h-BN substrate grown on $\operatorname{Ir}(111)$ are presented in Figure S1. Additional ARPES measurements of the Se-derive $\mathrm{p}_{z}$ states are shown in Figure S2. Figure S3 presents an overview of EDCs at the band minimum along $\bar{\Gamma}-\bar{K}$ and $\bar{\Gamma}-\bar{M}$ directions. The $\mathrm{CDW}$ in $\mathrm{SL}^{-\mathrm{NbSe}_{2}}$ grown on $\mathrm{WSe}_{2}$ is discussed by comparing the topography and FFT patterns of various different domains in Figure S4. The Van Hove singularities below $E_{\mathrm{F}}$ are discussed for $\mathrm{SL}-\mathrm{NbSe}_{2}$ on $\mathrm{BLG} / \mathrm{SiC}(0001)$ and for SL-NbSe ${ }_{2}$ on $\mathrm{h}-\mathrm{BN} / \mathrm{Ir}(111)$ in Figure S5. In Figure S6, we present further STS characterization with an applied magnetic field up to 5 $\mathrm{T}$, which confirms the superconducting character of the low-scale STS gap in SL-NbSe ${ }_{2}$ on h-BN/Ir(111). The same experiment has been conducted for $\mathrm{NbSe}_{2}$ on $\mathrm{WSe}_{2}$, which confirms the absence of SC in this system. By combining ARPES experiments with LEED measurements, the orientations of the SL-NbSe ${ }_{2}$ domains were studied and are presented in Figure S8 (PDF) 


\section{AUTHOR INFORMATION}

\section{Corresponding Authors}

Jill A. Miwa - Department of Physics and Astronomy, Interdisciplinary Nanoscience Center, Aarhus University, 8000 Aarhus C, Denmark; Email: miwa@phys.au.dk

Miguel M. Ugeda - Donostia International Physics Center (DIPC), 20018 San Sebastián, Spain; Centro de Física de Materiales (CSIC-UPV-EHU), 20018 San Sebastián, Spain; Ikerbasque, Basque Foundation for Science, 48013 Bilbao, Spain; $\odot$ orcid.org/0000-0001-7913-1617; Email:mmugeda@dipc.org

\section{Authors}

Paul Dreher - Donostia International Physics Center (DIPC), 20018 San Sebastián, Spain

Wen Wan - Donostia International Physics Center (DIPC), 20018 San Sebastián, Spain

Alla Chikina - Department of Physics and Astronomy, Interdisciplinary Nanoscience Center, Aarhus University, 8000 Aarhus C, Denmark

Marco Bianchi - Department of Physics and Astronomy, Interdisciplinary Nanoscience Center, Aarhus University, 8000 Aarhus C, Denmark

Haojie Guo - Departamento de Física de la Materia Condensada, Universidad Autonoma de Madrid, Madrid E28049, Spain; ๑ orcid.org/0000-0002-4407-0081

Rishav Harsh - Donostia International Physics Center (DIPC), 20018 San Sebastián, Spain

Samuel Mañas-Valero - Instituto de Ciencia Molecular (ICMol), Universitat de Valencia, 46980 Paterna, Spain

Eugenio Coronado - Instituto de Ciencia Molecular (ICMol), Universitat de Vatencia, 46980 Paterna, Spain; ๑ orcid.org/ 0000-0002-1848-8791

Antonio J. Martínez-Galera - Departamento de Física de la Materia Condensada, Universidad Autonoma de Madrid, Madrid E-28049, Spain; Insitituto Nicolás Cabrera, Universidad Autnoma de Madrid, Madrid E-28049, Spain; (- orcid.org/0000-0002-3982-7879

Philip Hofmann - Department of Physics and Astronomy, Interdisciplinary Nanoscience Center, Aarhus University, 8000 Aarhus C, Denmark; ๑ orcid.org/0000-0002-7367-5821

Complete contact information is available at:

https://pubs.acs.org/10.1021/acsnano.1c06012

\section{Author Contributions}

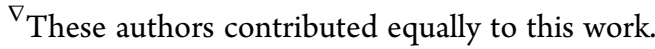

\section{Notes}

The authors declare no competing financial interest.

\section{ACKNOWLEDGMENTS}

We acknowledge fruitful discussions with Fernando de Juan, Félix Ynduráin, Carmen Rubio-Verdú, and Javier Zaldívar. We thank Marco Gobbi for carrying out check transport experiments. M.M.U. acknowledges support by the ERC Starting grant LINKSPM (grant 758558) and by the Spanish MINECO under grant no. PID2020-116619GB-C21. J.A.M. acknowledges financial support from the Danish Council for Independent Research, Natural Sciences under the Sapere Aude program (grant no. DFF-6108-00409). A.C, M.B., J.A.M., and P.H. acknowledge support from the VILLUM FONDEN via the Centre of Excellence for Dirac Materials (grant no. 11744). A.J.M.-G. acknowledges funding by the Spanish MINECO through project no. PID2020-116619GA-C22. E.C. and S.M.-V. acknowledge the Spanish MICINN (project PID2020117152RB-I00 cofinanced by FEDER and the Unit of Excellence "Maria de Maeztu" CEX2019-000919-M) and the Generalitat Valenciana (Prometeo Programme and PO FEDER Program IDIFEDER/2018/061 and IDFEDER/2020/063). R.H. acknowledges support from Marie Skłodowska-Curie Individual Fellowships under HORIZON 2020 program for project MAGTMD (101033538).

\section{REFERENCES}

(1) Chen, P.; Chan, Y.-H.; Fang, X.-Y.; Zhang, Y.; Chou, M. Y.; Mo, S.K.; Hussain, Z.; Fedorov, A.-V.; Chiang, T.-C. Charge Density Wave Transition in Single-Layer Titanium Diselenide. Nat. Commun. 2015, 6, 8943.

(2) Peng, J.-P.; Guan, J.-Q.; Zhang, H.-M.; Song, C.-L.; Wang, L.; He, K.; Xue, Q.-K.; Ma, X.-C. Molecular Beam Epitaxy Growth and Scanning Tunneling Microscopy Study of $\mathrm{TiSe}_{2}$ Ultrathin Films. Phys. Rev. B: Condens. Matter Mater. Phys. 2015, 91, 121113.

(3) Ugeda, M. M.; Bradley, A. J.; Zhang, Y.; Onishi, S.; Chen, Y.; Ruan, W.; Ojeda-Aristizabal, C.; Ryu, H.; Edmonds, M. T.; Tsai, H.-Z.; Riss, A.; Mo, S.-K.; Lee, D.; Zettl, A.; Hussain, Z.; Shen, Z.-X.; Crommie, M. F. Characterization of Collective Ground States in Single-Layer $\mathrm{NbSe}_{2}$. Nat. Phys. 2016, 12, 92-97.

(4) Ryu, H.; Chen, Y.; Kim, H.; Tsai, H.-Z.; Tang, S.; Jiang, J.; Liou, F.; Kahn, S.; Jia, C.; Omrani, A. A.; Shim, J. H.; Hussain, Z.; Shen, Z.-X.; Kim, K.; Min, B. I.; Hwang, C.; Crommie, M. F.; Mo, S.-K. Persistent Charge-Density-Wave Order in Single-Layer $\mathrm{TaSe}_{2}$. Nano Lett. 2018, $18,689-694$.

(5) Feng, J.; Biswas, D.; Rajan, A.; Watson, M. D.; Mazzola, F.; Clark, O. J.; Underwood, K.; Marković, I.; McLaren, M.; Hunter, A.; Burn, D. M.; Duffy, L. B.; Barua, S.; Balakrishnan, G.; Bertran, F.; le Fèvre, P.; Kim, T. K.; van der Laan, G.; Hesjedal, T.; Wahl, P.; King, P. D. C. Electronic Structure and Enhanced Charge-Density Wave Order of Monolayer $\mathrm{VSe}_{2}$. Nano Lett. 2018, 18, 4493-4499.

(6) Ye, J. T.; Zhang, Y. J.; Akashi, R.; Bahramy, M. S.; Arita, R.; Iwasa, Y. Superconducting Dome in a Gate-Tuned Band Insulator. Science 2012, 338, 1193-1196.

(7) Cao, Y.; Mishchenko, A.; Yu, G. L.; Khestanova, E.; Rooney, A. P.; Prestat, E.; Kretinin, A. V.; Blake, P.; Shalom, M. B.; Woods, C.; Chapman, J.; Balakrishnan, G.; Grigorieva, I. V.; Novoselov, K. S.; Piot, B. A.; Potemski, M.; Watanabe, K.; Taniguchi, T.; Haigh, S. J.; Geim, A. K.; Gorbachev, R. V. Quality Heterostructures from Two-Dimensional Crystals Unstable in Air by Their Assembly in Inert Atmosphere. Nano Lett. 2015, 15, 4914-4921.

(8) Xi, X.; Wang, Z.; Zhao, W.; Park, J.-H.; Law, K. T.; Berger, H.; Forró, L.; Shan, J.; Mak, K. F. Ising Pairing in Superconducting $\mathrm{NbSe}_{2}$ Atomic Layers. Nat. Phys. 2016, 12, 139-143.

(9) Zhao, K.; Lin, H.; Xiao, X.; Huang, W.; Yao, W.; Yan, M.; Xing, Y.; Zhang, Q.; Li, Z.-X.; Hoshino, S.; Wang, J.; Zhou, S.; Gu, L.; Bahramy, M. S.; Yao, H.; Nagaosa, N.; Xue, Q.-K.; Law, K. T.; Chen, X.; Ji, S.-H. Disorder-Induced Multifractal Superconductivity in Monolayer Niobium Dichalcogenides. Nat. Phys. 2019, 15, 904-910.

(10) Rubio-Verdu, C.; Garcia-Garcia, A. M.; Ryu, H.; Choi, D.-J.; Zaldıvar, J.; Tang, S.; Fan, B.; Shen, Z.-X.; Mo, S.-K.; Pascual, J. I.; Ugeda, M. M. Visualization of Multifractal Superconductivity in a TwoDimensional Transition Metal Dichalcogenide in the Weak-Disorder Regime. Nano Lett. 2020, 20, 5111-5118.

(11) Chen, Y.; Ruan, W.; Wu, M.; Tang, S.; Ryu, H.; Tsai, H.-Z.; Lee, R. L.; Kahn, S.; Liou, F.; Jia, C.; Albertini, O. R.; Xiong, H.; Jia, T.; Liu, Z.; Sobota, J. A.; Liu, A. Y.; Moore, J. E.; Shen, Z.-X.; Louie, S. G.; Mo, S.-K.; et al. Strong Correlations and Orbital Texture in Single-Layer 1T$\mathrm{TaSe}_{2}$. Nat. Phys. 2020, 16, 218-224.

(12) Bonilla, M.; Kolekar, S.; Ma, Y.; Diaz, H. C.; Kalappattil, V.; Das, R.; Eggers, T.; Gutierrez, H. R.; Phan, M.-H.; Batzill, M. Strong RoomTemperature Ferromagnetism in $\mathrm{VSe}_{2}$ Monolayers on van der Waals Substrates. Nat. Nanotechnol. 2018, 13, 289-293. 
(13) Fumega, A. O.; Gobbi, M.; Dreher, P.; Wan, W.; GonzálezOrellana, C.; Peña-Díaz, M.; Rogero, C.; Herrero-Martín, J.; Gargiani, P.; Ilyn, M.; Ugeda, M. M.; Pardo, V.; Blanco-Canosa, S. Absence of Ferromagnetism in $\mathrm{VSe}_{2}$ Caused by Its Charge Density Wave Phase. J. Phys. Chem. C 2019, 123, 27802-27810.

(14) Tang, S.; Zhang, C.; Wong, D.; Pedramrazi, Z.; Tsai, H.-Z.; Jia, C.; Moritz, B.; Claassen, M.; Ryu, H.; Kahn, S.; Jiang, J.; Yan, H.; Hashimoto, M.; Lu, D.; Moore, R. G.; Hwang, C.-C.; Hwang, C.; Hussain, Z.; Chen, Y.; Ugeda, M. M.; et al. Quantum Spin Hall State in Monolayer 1T'-WTe 2 . Nat. Phys. 2017, 13, 683-687.

(15) Fei, Z.; Palomaki, T.; Wu, S.; Zhao, W.; Cai, X.; Sun, B.; Nguyen, P.; Finney, J.; Xu, X.; Cobden, D. H. Edge Conduction in Monolayer WTe $e_{2}$. Nat. Phys. 2017, 13, 677-682.

(16) Ugeda, M. M.; Bradley, A. J.; Shi, S.-F.; da Jornada, F. H.; Zhang, Y.; Qiu, D. Y.; Ruan, W.; Mo, S.-K.; Hussain, Z.; Shen, Z.-X.; Wang, F.; Louie, S. G.; Crommie, M. F. Giant Bandgap Renormalization and Excitonic Effects in a Monolayer Transition Metal Dichalcogenide Semiconductor. Nat. Mater. 2014, 13, 1091-1095.

(17) Shao, B.; Eich, A.; Sanders, C.; Ngankeu, A. S.; Bianchi, M.; Hofmann, P.; Khajetoorians, A. A.; Wehling, T. O. Pseudodoping of a Metallic Two-Dimensional Material by the Supporting Substrate. Nat. Commun. 2019, 10, 180.

(18) Dendzik, M.; Bruix, A.; Michiardi, M.; Ngankeu, A. S.; Bianchi, M.; Miwa, J. A.; Hammer, B.; Hofmann, P.; Sanders, C. E. SubstrateInduced Semiconductor-to-Metal Transition in Monolayer $\mathrm{WS}_{2}$. Phys. Rev. B: Condens. Matter Mater. Phys. 2017, 96, 235440.

(19) Duvjir, G.; Choi, B. K.; Ly, T. T.; Lam, N. H.; Jang, K.; Dung, D. D.; Chang, Y. J.; Kim, J. Multiple Charge Density Wave Phases of Monolayer $\mathrm{VSe}_{2}$ Manifested by Graphene Substrates. Nanotechnology 2021, 32, 364002.

(20) Zhang, C.; Li, M.-Y.; Tersoff, J.; Han, Y.; Su, Y.; Li, L.-J.; Muller, D. A.; Shih, C.-K. Strain Distributions and Their Influence on Electronic Structures of $\mathrm{WSe}_{2}-\mathrm{MoS}_{2}$ Laterally Strained Heterojunctions. Nat. Nanotechnol. 2018, 13, 152-158.

(21) Gao, S.; Flicker, F.; Sankar, R.; Zhao, H.; Ren, Z.; Rachmilowitz, B.; Balachandar, S.; Chou, F.; Burch, K. S.; Wang, Z.; van Wezel, J.; Zeljkovic, I. Atomic-Scale Strain Manipulation of a Charge Density Wave. Proc. Natl. Acad. Sci. U. S. A. 2018, 115, 6986-6990.

(22) Sanders, C. E.; Dendzik, M.; Ngankeu, A. S.; Eich, A.; Bruix, A.; Bianchi, M.; Miwa, J. A.; Hammer, B.; Khajetoorians, A. A.; Hofmann, P. Crystalline and Electronic Structure of Single-Layer $\mathrm{TaS}_{2}$. Phys. Rev. B: Condens. Matter Mater. Phys. 2016, 94, 081404.

(23) Cheng, F.; Ding, Z.; Xu, H.; Tan, S. J. R.; Abdelwahab, I.; Su, J.; Zhou, P.; Martin, J.; Loh, K. P. Epitaxial Growth of Single-Layer Niobium Selenides with Controlled Stoichiometric Phases. Adv. Mater. Interfaces 2018, 5, 1800429.

(24) Stan, R.-M.; Mahatha, S. K.; Bianchi, M.; Sanders, C. E.; Curcio, D.; Hofmann, P.; Miwa, J. A. Epitaxial Single-Layer $\mathrm{NbS}_{2}$ on $\mathrm{Au}(111)$ : Synthesis, Structure, and Electronic Properties. Phys. Rev. Materials 2019, 3, 044003.

(25) Lefcochilos-Fogelquist, H. M.; Albertini, O. R.; Liu, A. Y. Substrate-Induced Suppression of Charge Density Wave Phase in Monolayer $1 \mathrm{H}-\mathrm{TaS}_{2}$ on $\mathrm{Au}(111)$. Phys. Rev. B: Condens. Matter Mater. Phys. 2019, 99, 174113.

(26) Grønborg, S. S.; Ulstrup, S.; Bianchi, M.; Dendzik, M.; Sanders, C. E.; Lauritsen, J. V.; Hofmann, P.; Miwa, J. A. Synthesis of Epitaxial Single-Layer $\mathrm{MoS}_{2}$ on $\mathrm{Au}(111)$. Langmuir 2015, 31, 9700-9706.

(27) Lin, H.; Huang, W.; Zhao, K.; Lian, C.; Duan, W.; Chen, X.; Ji, S.H. Growth of Atomically Thick Transition Metal Sulfide Films on Graphene/6H-SiC (0001) by Molecular Beam Epitaxy. Nano Res. 2018, 11, 4722-4727.

(28) Hall, J.; Ehlen, N.; Berges, J.; van Loon, E.; van Efferen, C.; Murray, C.; Rösner, M.; Li, J.; Senkovskiy, B. V.; Hell, M.; Rolf, M.; Heider, T.; Asensio, M. C.; Avila, J.; Plucinski, L.; Wehling, T.; Grüneis, A.; Michely, T. Environmental Control of Charge Density Wave Order in Monolayer $2 \mathrm{H}-\mathrm{TaS}_{2}$. ACS Nano 2019, 13, 10210-10220.

(29) Calandra, M.; Mazin, I. I.; Mauri, F. Effect of Dimensionality on the Charge-Density Wave in Few-Layer $2 \mathrm{H}-\mathrm{NbSe}_{2}$. Phys. Rev. B: Condens. Matter Mater. Phys. 2009, 80, 241108.
(30) Silva-Guillén, J. Á.; Ordejón, P.; Guinea, F.; Canadell, E. Electronic Structure of $2 \mathrm{H}-\mathrm{NbSe}_{2}$ Single-Layers in the CDW State. $2 \mathrm{D}$ Mater. 2016, 3, 035028.

(31) Blase, X.; Rubio, A.; Louie, S. G.; Cohen, M. L. Quasiparticle Band Structure of Bulk Hexagonal Boron Nitride and Related Systems. Phys. Rev. B: Condens. Matter Mater. Phys. 1995, 51, 6868-6875.

(32) Brihuega, I.; Michaelis, C. H.; Zhang, J.; Bose, S.; Sessi, V.; Honolka, J.; Alexander Schneider, M.; Enders, A.; Kern, K. Electronic Decoupling and Templating of Co Nanocluster Arrays on the Boron Nitride Nanomesh. Surf. Sci. 2008, 602, L95-L99.

(33) Bose, S.; García-García, A. M.; Ugeda, M. M.; Urbina, J. D.; Michaelis, C. H.; Brihuega, I.; Kern, K. Observation of Shell Effects in Superconducting Nanoparticles of Sn. Nat. Mater. 2010, 9, 550-554.

(34) Auwärter, W. Hexagonal Boron Nitride Monolayers on Metal Supports: Versatile Templates for Atoms, Molecules and Nanostructures. Surf. Sci. Rep. 2019, 74, 1-95.

(35) Wan, W.; Dreher, P.; Muñoz-Segovia, D.; Harsh, R.; Guinea, F.; de Juan, F.; Ugeda, M. M. Observation of Superconducting Leggett Modes from Competing Pairing Instabilities in Single-Layer $\mathrm{NbSe}_{2}$. arXiv (Superconductivity), September 15, 2021, 2101.04050v2, ver. 2. https://arxiv.org/abs/2101.04050v2 (accessed 2021-09-15).

(36) Schulz, F.; Drost, R.; Hämäläinen, S. K.; Demonchaux, T.; Seitsonen, A. P.; Liljeroth, P. Epitaxial Hexagonal Boron Nitride on $\operatorname{Ir}(111)$ : A Work Function Template. Phys. Rev. B: Condens. Matter Mater. Phys. 2014, 89, 235429.

(37) Miwa, J. A.; Ulstrup, S.; Sørensen, S. G.; Dendzik, M.; Cabo, A. G.; Bianchi, M.; Lauritsen, J. V.; Hofmann, P. Electronic Structure of Epitaxial Single-Layer $\mathrm{MoS}_{2}$. Phys. Rev. Lett. 2015, 114, 046802.

(38) Shao, B.; Eich, A.; Sanders, C.; Ngankeu, A. S.; Bianchi, M.; Hofmann, P.; Khajetoorians, A. A.; Wehling, T. O. Pseudodoping of a Metallic Two-Dimensional Material by the Supporting Substrate. Nat. Commun. 2019, 10, 180.

(39) DE Gennes, P. G. Boundary Effects in Superconductors. Rev. Mod. Phys. 1964, 36, 225-237.

(40) Riley, J. M.; Mazzola, F.; Dendzik, M.; Michiardi, M.; Takayama, T.; Bawden, L.; Granerød, C.; Leandersson, M.; Balasubramanian, T.; Hoesch, M.; Kim, T. K.; Takagi, H.; Meevasana, W.; Hofmann, Ph.; Bahramy, M. S.; Wells, J. W.; King, P. D. C. Direct Observation of SpinPolarized Bulk Bands in an Inversion-Symmetric Semiconductor. Nat. Phys. 2014, 10, 835-839.

(41) Wan, W.; Dreher, P.; Harsh, R.; Ugeda, M. M. Manuscript in preparation, 2021.

(42) Chikina, A.; Fedorov, A.; Bhoi, D.; Voroshnin, V.; Haubold, E.; Kushnirenko, Y.; Kim, K. H.; Borisenko, S. Turning Charge-Density Waves into Cooper Pairs. npj Quantum Materials 2020, 5, 22.

(43) Horcas, I.; Fernández, R.; Gómez-Rodríguez, J. M.; Colchero, J.; Gómez-Herrero, J.; Baró, A. M. WSxM: A Software for Scanning Probe Microscopy and a Tool for Nanotechnology. Rev. Sci. Instrum. 2007, 78, 013705 .

(44) Hoffmann, S.; Søndergaard, C.; Schultz, C.; Li, Z.; Hofmann, P. An Undulator-based Spherical Grating Monochromator Beamline for Angle-Resolved Photoemission Spectroscopy. Nucl. Instrum. Methods Phys. Res., Sect. A 2004, 523, 441-453. 